\title{
CONVEX HULLS OF SIMPLE SPACE GURVES
}

\author{
DOUGLAS DERRY
}

1. Introduction. The convex hull of an arbitrary set $M$ in real Euclidean $n$-space is known to consist of all the points within the $r$-simplexes with $r+1$ vertices from $M, r \leqslant n$. This note shows that if $M$ is specialized to be a curve $A_{n}$ of real order $n$, then its convex hull consists of all the points within the $r$-simplexes with $r+1$ vertices on $A_{n}, n=2 r+1$ or $n=2 r$. In the first case each interior point is within exactly one simplex. This result was given by Egerváry (1) for $n=3$. If $n$ is even each interior point of the convex hull of $A_{n}$ is within a 1 -parameter system of $\frac{1}{2} n$-simplexes. The class of curves $A_{n}$ includes the twisted $n$-ics, the convex hulls of which have been studied by Karlin and Shapley (2). Some of their results are consequences of the present results.

2. Some definitions. A curve $A_{n}$ is defined to be a 1-1 continuous mapping in real Euclidean $n$-space of all the real numbers $s$ computed modulo 1 or of the interval, $0 \leqslant s \leqslant 1$, which satisfies the order condition that no hyperplane contains more than $n$ points of $A_{n}$.

The order condition implies that any linear $k$-space, $0 \leqslant k<n$, cannot contain more than $k+1$ points of $A_{n}$. If a hyperplane $H$ supports $A_{n}$ at an inner point $s^{\prime}$ then $s^{\prime}$ is defined to have multiplicity two within $H$. By displacing the hyperplanes it is possible to show that the sharpened order condition ${ }^{1}$ holds that no hyperplane contains more than $n$ points of $A_{n}$ if each point is counted with its proper multiplicity of one or two.

The symbol $[A, B, \ldots]$ denotes the intersection of all the linear spaces which include the point sets $A, B, \ldots$, while $\{A, B, \ldots\}$ denotes the convex hull of the union of the point sets $A, B, \ldots$ Two sets $A$ and $B$ are said to be separated by a hyperplane $H$ provided $A$ is in one of the closed half spaces bounded by $H$ and $B$ in the other.

3. The boundary of $A_{n}$. The following lemma is stated without proof.

Lemma 1. If a hyperplane $H$ supports a compact set $X$, then $\{H \cap X\}=$ $H \cap\{X\}$.

THEOREM 1. The boundary of $\left\{A_{n}\right\}$ consists of all the points within all the $q$-simplexes for which the vertices are $q+1$ points of $A_{n}$ including e endpoints, $2 q \leqslant n-2+e,(e=0,1,2)$.

Received October 14, 1955.

${ }^{1}$ I should like to thank the referee for the improvements he suggested and in particular for pointing out that the above form of the sharpened order condition, which makes no use of differentiability, was sufficient for the results of this paper. 
Proof. If $P$ be a boundary point of $\left\{A_{n}\right\}$, a hyperplane $H$ exists which supports $A_{n}$ and contains $P$. Let $s_{0}, s_{1}, \ldots, s_{q}$ be the distinct curve points in $H \cap\left\{A_{n}\right\}$. Because of the order condition, $\left\{s_{0}, s_{1}, \ldots, s_{q}\right\}$ is a $q$-simplex. By Lemma 1 ,

$$
P \in H \cap\left\{A_{n}\right\}=\left\{H \cap A_{n}\right\}=\left\{s_{0}, s_{1}, \ldots, s_{q}\right\} .
$$

As $H$ supports $A_{n}$ an interior point $s_{i}$ of $A_{n}$ must be included in $H$ twice. Consequently if $e$ denotes the number of endpoints of $A_{n}$ in $H$, it follows from the order condition that

$$
e+2(q+1-e) \leqslant n \text { or } 2 q \leqslant n-2+e .
$$

Thus each boundary point of $\left\{A_{n}\right\}$ is within a $q$-simplex, with the required properties.

Conversely let $P$ be a point of a $q$-simplex $\left\{s_{0}, s_{1}, \ldots, s_{q}\right\}$ for which $2 q \leqslant n-$ $2+e$. Then a hyperplane exists which contains $P$ and supports $A_{n}$. To construct such a hyperplane, for each point $s_{i}$ interior to $A_{n}$, let $N_{i}$ be an arc $s^{\prime}{ }_{i}<s<s_{i}$ and if $A_{n}$ is not closed let $N^{\prime}, N^{\prime \prime}$ be neighbourhoods of the endpoints 0,1 respectively. Let $H$ be a hyperplane which contains $n$ points of $A_{n}$ including all $s_{i}, s^{\prime}{ }_{i}$ and so that the remaining $n-2(q+1)+e$ curve points within $H$ are distributed among the $\operatorname{arcs} N_{i}, N^{\prime}, N^{\prime \prime}$ in such a way that no arc $N_{i}$ contains an odd number of these points. This distribution is always possible because if $A_{n}$ is closed $n$ is even and $e=0$. If $N_{i} \rightarrow s_{i}, N^{\prime} \rightarrow 0$, $N^{\prime \prime} \rightarrow 1$ then any limiting position of $H$ contains $P$ and supports $A_{n}$. As $P \in\left\{s_{0}, s_{1}, \ldots, s_{q}\right\} \subseteq\left\{A_{n}\right\}, P$ is a boundary point of $\left\{A_{n}\right\}$. The proof is now complete.

4. The structure of $\left\{A_{n}\right\}$. If $2 r=n$ or $2 r+1=n, S_{r}$ is defined to be an $r$-simplex with interior points of $A_{n}$ as vertices except for even $n$ when at most one of the vertices may be an endpoint of $A_{n}$.

Theorem 2. The interior points $P$ of $\left\{A_{n}\right\}$ consist of all the interior points of the simplexes $S_{r}$.

For odd $n, S_{r}$ is uniquely determined by any one of its interior points $P$; for even $n, S_{r}$ is uniquely determined by an interior point $P$ and any one vertex which can be either endpoint of $A_{n}$ or any arbitrary point of $A_{n}$ if it is closed.

Proof. We show first that every interior point $P$ of a simplex $S_{r}$ is an interior point of $\left\{A_{n}\right\}$. As $S_{r} \subseteq\left\{A_{n}\right\}$ it will be sufficient to show $P$ is not a boundary point of $\left\{A_{n}\right\}$. Let $e$ be the number of vertices of $S_{r}$ which are endpoints of $A_{n}$. If $P$ were a boundary point of $\left\{A_{n}\right\}$ it would be within a hyperplane $H$ which would support $\left\{A_{n}\right\}$. $H$ would also support $S_{r}$ and consequently, as $P$ is an inner point of $S_{r}, S_{r} \subseteq H$. Therefore $H$ would contain $2(r+1-e)+e$ points of $A_{n}$. This would contradict the order condition as, by the definition of $S_{r}, e=0$ if $n=2 r+1$ and $e \leqslant 1$ if $n=2 r$. Hence the inner points of the simplexes $S_{r}$ are all inner points of $\left\{A_{n}\right\}$. 
We next show that a given interior point $P$ of $\left\{A_{n}\right\}$ is an interior point of a simplex $S_{r}$. Let $a$ be any real number if $A_{n}$ is closed and 0 if $A_{n}$ is open. Denote by $A\left(a, s^{\prime}\right)$ the arc of points $s, a \leqslant s \leqslant s^{\prime}$. Let $s_{P}$ be the least upper bound of all $s^{\prime}$ for which $P \notin\left\{A\left(a, s^{\prime}\right)\right\}$.

We prove that $P \in\left\{A\left(a, s_{P}\right)\right\}$. If this were false, $P$ and $\left\{A\left(a, s_{P}\right)\right\}$ would be separated by a hyperplane at a positive distance from $\left\{A\left(a, s_{P}\right)\right\}$. This hyperplane would also separate $A\left(a, s^{\prime}\right)$ and $P$ for $s^{\prime}>s_{P}$ provided $s^{\prime}$ were sufficiently close to $s_{P}$. Consequently $P \notin\left\{A\left(a, s^{\prime}\right)\right\}$ contrary to the choice of $s_{P}$.

$P$ is on a supporting hyperplane of $\left\{A\left(a, s_{P}\right)\right\}$. To prove this let $s_{\mu}$ be an increasing sequence which converges to $s_{P}$. Because $P \notin\left\{A\left(a, s_{\mu}\right)\right\}$ a hyperplane $H_{\mu}$ exists which supports $\left\{A\left(a, s_{\mu}\right)\right\}$ and contains $P . s_{\mu}$ can be chosen so that $H_{\mu}$ converges. If $H$ be its limit then $P \in H$ and $H$ supports $\left\{A\left(a, s_{\mu}\right)\right\}$. But, as $s_{\mu}$ is arbitrary, $H$ supports $\left\{A\left(a, s_{P}\right)\right\}$. From this result, together with the fact that $P \in\left\{A\left(a, s_{P}\right)\right\}$, it follows that $P$ is a boundary point of $\left\{A\left(a, s_{P}\right)\right\}$.

Consequently, by Theorem 1 , a simplex $S_{q}$ exists which contains $P$, has vertices on $A\left(a, s_{P}\right)$ and for which $2 q \leqslant n-2+e$, where $e$ is the number of vertices of $S_{q}$ which are endpoints of $A\left(a, s_{P}\right)$. The vertices of $S_{q}$ are also on $A_{n}$. Let $e^{\prime}$ be the number of these vertices which are endpoints of $A_{n}$. As $P$ is not a boundary point of $\left\{A_{n}\right\}, 2 q>n-2+e^{\prime}$. Therefore $e^{\prime}<e$ and so $0<e$. If $A_{n}$ is open, $e^{\prime}=e-1$ as 0 is a common endpoint of $A_{n}$ and $A\left(a, s_{P}\right)$. The two inequalities yield the result $2 q=n-2+e$. Hence, if $n=2 r$, then $e=2$ and $q=r$ and, if $n=2 r+1, e=1$ and $q=r$. If $A_{n}$ is closed $n$ is even and $e^{\prime}=0$. In this case the inequalities show $e=2$ and $r=q$. $P$ cannot be a point of a face of $S_{r}$ for such points, by Theorem 1 , are boundary points of $\left\{A_{r}\right\}$. Therefore $P$ is an interior point of the $r$-simplex $S_{r}$ which satisfies the requirements of the theorem as $e^{\prime}=0$ for odd $n$ and $e^{\prime} \leqslant 1$ for even $n$. This completes the proof of the first part of the theorem.

For even $n, e=2$ and consequently $a$ is a vertex of $S_{r}$. If $A_{n}$ is closed $a$ is arbitrary and so in this case, for a given $P$, an $S_{r}$ exists with an arbitrary vertex. If $A_{n}$ is open $a=0$. After a reversal of orientation of the points on the curve, the other endpoint of $A_{n}$ can be represented by the number 0 . Therefore $S_{r}$ can be chosen so that either endpoint of $A_{n}$ is a vertex provided $n$ is even.

Suppose now $P$ is a point within two distinct simplexes with vertices $s_{0}, s_{1}, \ldots, s_{r} ; s^{\prime}{ }_{0}, s^{\prime}{ }_{1}, \ldots, s^{\prime}{ }_{r}$ and that $P$ is not in a face of $\left\{s_{0}, s_{1}, \ldots, s_{r}\right\}$. Let $k, 0 \leqslant k \leqslant r$, be the number of vertices common to both simplexes. It follows, with the use of the Steinitz replacement theorem, that the space

$$
\left[s_{0}, s_{1}, \ldots, s_{r}, s^{\prime}{ }_{0}, s^{\prime}{ }_{1}, \ldots, s_{r}^{\prime}{ }_{r}\right]
$$

has dimension at most $2 r-k$. It contains $2(r+1)-k$ points of $A_{n}$. This leads to a contradiction of the order condition unless $2 r-k=n$ in which case $k=0$ and $n=2 r$. This proves, for odd $n$, that $P$ is within only one simplex $S_{r}$ and, for even $n$, that $P$ is never in more than one simplex $S_{r}$ with a given vertex. The proof is now complete. 
CoRollary. Every point $P$ in the interior of $\left\{A_{2 r}\right\}$ is an interior point of each of two suitably chosen simplexes $S_{r}, S^{\prime}{ }_{r}$ which have no common vertex.

Proof. If $A_{2 r}$ is open each interior point $P$ of $\left\{A_{2 r}\right\}$ is, by the Theorem, interior to a simplex $S_{r}\left(S^{\prime}{ }_{r}\right)$ with the endpoint $s=0,(s=1)$ as a vertex. If $S_{r}, S^{\prime}{ }_{r}$ were to have a common vertex then, by the Theorem, they would be identical and both endpoints of $A_{2 r}$ would be vertices in contradiction to the definition of the simplexes. If $A_{2 r}$ is closed the result is clear.

Lemma 2. If the vertices of two $r$-simplexes $S_{r}, S^{\prime}{ }_{r}$ which have no common vertex are all on $A_{2_{r}}$ and if an arc of $A_{2_{r}}$ exists which contains two vertices of $S_{r}$ and no vertex of $S^{\prime}{ }_{r}$, then $S_{r}, S_{r}{ }_{r}$ have no point in common.

Proof. Let $s_{0}, s_{1}, \ldots, s_{r}, s_{0}<s_{1}<\ldots<s_{r}<s_{0}+1 \quad\left(=s_{r+1}\right)$ be the vertices of $S_{r}$. By the hypothesis an arc $s_{k} \leqslant s \leqslant s_{k+1}$ exists which contains no vertex of $S_{r}^{\prime}, 0 \leqslant k<r$, if $A_{2 r}$ is open and $0 \leqslant k \leqslant r$, if $A_{2 r}$ is closed. In the latter case the coordinates may be adjusted so that $0 \leqslant k<r$. As $S_{r}, S^{\prime}{ }_{r}$ have no common vertex, distinct curve points $t^{\prime}{ }_{1}, t_{1}, \ldots, t^{\prime}{ }_{r}, t_{r}$ of $A_{2 r}$ exist so that

$$
\begin{gathered}
t^{\prime}{ }_{1} \leqslant s_{0} \leqslant t_{1}<t^{\prime}{ }_{2} \leqslant s_{2} \leqslant t_{2}<\ldots<t^{\prime}{ }_{k+1} \leqslant s_{k}<s_{k+1} \leqslant t_{k+1}<\ldots \\
<t^{\prime}{ }_{r} \leqslant s_{r} \leqslant t_{r} \leqslant t^{\prime}{ }_{1}+1
\end{gathered}
$$

and so that none of the $\operatorname{arcs} t^{\prime}{ }_{1} \leqslant s \leqslant t_{i}, 1 \leqslant i \leqslant r$, contains a vertex of $S^{\prime}{ }_{r}$. Let $H$ be the hyperplane $\left[t^{\prime}{ }_{1}, t_{1}, \ldots, t^{\prime}{ }_{r}, t_{r}\right]$. As $H$ intersects $A_{2 r}$ only in the $2 r$ points $t^{\prime}{ }_{i}, t_{i}, 1 \leqslant i \leqslant r$, all the points of the $\operatorname{arcs} t^{\prime}{ }_{i} \leqslant s \leqslant t_{i}, 1 \leqslant i \leqslant r$, are either on $H$ or on the same side of $H$ while all the points of $A_{2 r}$ not within the above arcs are on the opposite side of $H$. Thus $H$ separates the vertices of $S_{r}$ from those of $S^{\prime}{ }_{r}$. Furthermore all the vertices of $S^{\prime}{ }_{r}$ are at a positive distance from $H$. Hence $S_{r}$ and $S_{r}^{\prime}$ have no points in common. The Lemma is now proved.

Convex hulls are defined for affine space. The following result shows that the convex hull $\left\{A_{2 r}\right\}$ can be defined in terms of projective concepts.

Theorem 3. If $s_{0}, s_{1}, \ldots, s_{r} ; s^{\prime}{ }_{0}, s^{\prime}{ }_{1}, \ldots, s_{r}^{\prime}$ are curve points of $A_{2 r}$ for which

$$
0 \leqslant s_{0}<s_{0}^{\prime}<s_{1}<\ldots<s_{r}<s_{r}^{\prime} \leqslant 1,
$$

for open $A_{2 r}$ and

$$
s_{0}<s_{0}^{\prime}<s_{1}<\ldots<s_{r}<s^{\prime}{ }_{r}<s_{0}+1\left(=s_{r+1}\right)
$$

for closed $A_{2 r}$ then the interior of $\left\{A_{2 r}\right\}$ consists of all the intersections

$$
\left[s_{0}, s_{1}, \ldots, s_{r}\right] \cap\left[s^{\prime}{ }_{0}, s^{\prime}{ }_{1}, \ldots, s^{\prime}{ }_{r}\right] .
$$

Proof. Let $P$ be a given point in the interior of $\left\{A_{2 r}\right\}$. By the Corollary to Theorem 2 , simplexes $S_{r}, S^{\prime}{ }_{r}$ exist, without a common vertex, both of which 
contain $P$ as an interior point. Let $s_{0}, s_{1}, \ldots, s_{r}, 0 \leqslant s_{0}<s_{1}<\ldots<s_{r}<$ $s_{0}+1$ be the vertices of $S_{r}$. As $S_{r}, S^{\prime}{ }_{r}$ have the common interior point $P$ it follows from Lemma 2 that each arc $s_{i} \leqslant s \leqslant s_{i+1}, 0 \leqslant i<r$, contains exactly one vertex of $S^{\prime}{ }_{r}$. Therefore if $s^{\prime}{ }_{0}, s^{\prime}{ }_{1}, \ldots, s^{\prime}{ }_{r}$ be the vertices of $S^{\prime}$ r the subscripts may be adjusted so that, for closed $A_{2 r}$,

$$
s_{0}<s_{0}^{\prime}<s_{1}<\ldots<s^{\prime}{ }_{r-1}<s_{\tau}<s^{\prime}{ }_{r}<s_{0}+1
$$

and, for open $A_{2 r}$, either

$$
0 \leqslant s_{0}<s^{\prime}{ }_{0}<s_{1}<\ldots<s_{r}<s^{\prime}{ }_{r} \leqslant 1
$$

or

$$
0 \leqslant s^{\prime}{ }_{0}<s_{0}<\ldots<s^{\prime}{ }_{r}<s_{r} \leqslant 1 .
$$

As $P$ is a common point of the simplexes

$$
P \in\left[s_{0}, s_{1}, \ldots, s_{r}\right] \cap\left[{s^{\prime}}_{0}, s^{\prime}{ }_{1}, \ldots, s^{\prime}{ }_{r}\right] .
$$

Now let $Q$ be any point of $\left[s_{0}, s_{1}, \ldots, s_{r}\right] \cap\left[s^{\prime}{ }_{0}, s^{\prime}{ }_{1}, \ldots, s^{\prime}{ }_{r}\right]$ where

$$
s_{0}, s_{1}, \ldots, s_{r}, s^{\prime}{ }_{0}, s_{1}^{\prime}, \ldots, s_{r}^{\prime}
$$

are points of $A_{2 r}$ which satisfy the inequality system. The $r$-spaces $\left[s_{0}, s_{1}\right.$, $\left.\ldots, s_{r}\right],\left[s^{\prime}{ }_{0}, s^{\prime}{ }_{1}, \ldots, s^{\prime}{ }_{r}\right]$ must have at least one point in common as $2 r=n$. They cannot have more than one point in common for then

$$
\left[s_{0}, s_{1}, \ldots, s_{r}, s_{0}^{\prime}, \ldots, s_{r}{ }_{r}\right]
$$

would have dimension at most $2 r-1$ and contain $2 r+2$ points of $A_{2 r}$, in contradiction to the order condition.

$Q$ cannot be a point on a proper face of either simplex $\left\{s_{0}, s_{1}, \ldots, s_{r}\right\}$, $\left\{s^{\prime}{ }_{0}, s^{\prime}{ }_{1}, \ldots, s_{r}{ }_{r}\right\}$. Suppose, for example, $Q$ to be within the face $\left\{s_{0}, s_{1}, \ldots\right.$, $\left.s_{r-1}\right\}$. Then the space

$$
\left[s_{0}, s_{1}, \ldots, s_{r-1}, s^{\prime}{ }_{0}, \ldots, s^{\prime}{ }_{r}\right]
$$

would have dimension at most $2 r-1$ and contain $2 r+1$ points of $A_{2 r}$ in contradiction to the order condition.

If $s_{0}, s_{1}, \ldots, s_{r}, s^{\prime}{ }_{0}, \ldots, s^{\prime}{ }_{r}$ move continuously so that the inequalities are always satisfied, $Q$ is uniquely defined and moves continuously. We know, if $Q=P$, that $Q$ is interior to $\left\{A_{2 r}\right\}$ as well as to both simplexes $\left\{s_{0}, s_{1}, \ldots, s_{r}\right\}$, $\left\{s^{\prime}{ }_{0}, s^{\prime}{ }_{1}, \ldots, s^{\prime}{ }_{r}\right\}$. As $Q$ cannot enter a proper face of either of these simplexes it must remain in the interior of both of them. $Q$ cannot enter the boundary of $\left\{A_{2 r}\right\}$. For otherwise it would be in a hyperplane $H$ supporting $\left\{A_{2 r}\right\}$ and consequently supporting $\left\{s_{0}, s_{1}, \ldots, s_{r}\right\}$. As $Q$ is an interior point of the simplex, $\left[s_{0}, s_{1}, \ldots, s_{r}\right] \subseteq H$. It follows from the inequality system that at most one vertex of $\left\{s_{0}, s_{1}, \ldots, s_{r}\right\}$ is an endpoint of $A_{2 r}$. Hence $H$ would contain at least $2(r+1)-1=2 r+1$ points of $A_{2 r}$ in contradiction to the order condition. Therefore $Q$ must always remain in the interior of $\left\{A_{2 r}\right\}$. The proof is now complete. 


\section{REFERENCES}

1. E. Egerváry, On the smallest cover of a simple arc of space curve. Publ. Math. Debrecen 1 (1949), 65-70.

2. S. Karlin and L. S. Shapley, Geometry of moment spaces. Mem. Amer. Math. Soc. 12 (1953).

\section{University of British Columbia}

